\title{
A Persona-based Modelling for Contextual Requirements
}

\author{
Genaína Nunes Rodrigues ${ }^{1}$, Carlos Joel Tavares ${ }^{1}$, Naiara Watanabe ${ }^{1}$, \\ Carina Alves ${ }^{2}$, and Raian $\mathrm{Ali}^{3}$ \\ 1 University of Brasília, Brazil \\ genaina@unb.br, carlosjoel.tavares@gmail.com, naiara@gmail.com \\ ${ }^{2}$ Federal University of Pernambuco, Center of Informatics, Brazil \\ cfa@cin.ufpe.br \\ 3 Bournemouth University, UK \\ rali@bournemouth.ac.uk
}

\begin{abstract}
Context\&Motivation] Personas are a technique used to guide developing products accommodating people diversity. They are archetypes reflecting common combinations of users' characteristics, needs and goals. Persons can add a human-centred facet to requirements engineering practice which is often revolving around the concept of business roles. [Question/Problem] Goal modelling is an example of mainstream requirements engineering approach driven by business roles and their responsibilities and needs represented as goals. Personnel in the system are expected to act according to this prescriptive specification. Personnel diversity is often seen as a customization and design issue. [Principal idea/Results]. In this paper we propose to consider such diversity as a conditional context in requirements modelling and, as an approach, augment Contextual Goal Model (CGM) with personas as a new contextual dimension. Additionally, we propose an algorithm to analyse the achievability of CGM goals in the presence of the personas contexts variation. We evaluate our approach using a Mobile Personal Emergency Response System (MPERS) implemented as a prototype. [Contribution] Our persona-based modelling approach paves the way to augment requirements with a consideration of people diversity and enrich the business perspective with a more user-centred design facet.
\end{abstract}

Keywords: Contextual Requirements, User-Centred Design, Goal-Oriented Requirements Engineering

\section{Introduction}

A persona is a fictional character that represents a group of users of a given system. It is a design technique used in product development that complements other usability techniques, rendering the product development more effective and accommodative to diversity [5[16]. The use of personas puts a face on the user, making them as real as possible and helping in that sense making the design more human-centred. The personas are defined by their attributes, goals and any other information that might help the development process (e.g. age, skills, tasks, etc). Such attributes and goals guide the 
development of a system solution and may provide a significant advantage during the research and conceptualisation stages of the design process [13].

Goal-oriented requirements engineering (GORE) uses goals for the elaboration, specification, negotiation, documentation and modification of requirements in system development [18]. Goal models (GM) provide the goals for which the system should be designed and a set of ways to reach those goals in prescriptive and pragmatic manners [15[20]. Personas, on the other hand, are synthesized into descriptions that include behavior patterns, goals, skills/capabilities, attitudes, and environment [5]. Therefore, goals and capabilities are core and also shared constituents for both goal modelling and personas making the integration of power between both techniques easier and natural.

GORE in general tends to take a business perspective where people are allocated to roles, responsibilities and permissions. However, we recognize that the individual levels cannot be normalized and would not be expected to play the same role in a similar and uniform way. On the other hand, the consideration of the personal differences case by case adds infeasible overhead to the engineers and introduce the need for personalizing the requirements [17]. We propose the use of personas as a feasible mechanism putting two perspectives together. First, as a way to handle the lack of consideration of users as people with personality and goals not necessarily completely aligned with their roles in the system. Second, as a normative way of operation to handle the complexity of taking into account a multitude of personal difference.

Persona information can be modelled as contextual conditions on the set of requirements and their alternative strategies of achievement following from their behavior patterns and capabilities. By context we mean a partial state of the world that system operates [1] specified as a formula or world predicate that must be fulfilled to enable the activation of a requirement and also its achievement through certain alternative pathways in the goal analysis. For example, consider a system that requires a person to specify their location and situation in case they require a prompt delivery of an ambulance. If the person is averse to technology, they might not be able to perform well such action in such emergency situation. However, such aversion is not exactly a personal goal or neither a system goal, but instead part of the person's characteristic that enables or hinders the need for certain goals and their alternative ways of achievements.

In this paper, we introduce the notion of personas as a source of context when modelling contextual requirements so that we empower requirements modelling practice with personalization and human-centred design facets. In addition, we propose a method for goal achievement sensitive to their actual set of personas. We formalize a structure where personas attributes and goals are then used to define the contextual operation the system. Therefore, the contributions of the paper are threefold. First, we propose a methodology that articulates the information of personas and map them to contextual goal models (CGM) [1] as contextual condition. Secondly, we propose an algorithm to analyse the achievability of CGM goals in the presence of the personas contexts variation. Lastly, we carry out an exploratory study performed on the Mobile Personal Emergency System (MPERS) [15] to assess the benefits and feasibility of the approach.

The remaining sections of the paper are structured as follows: Section 2 explores the background needed for understanding the paper. Section 3 we present our conceptual model and the methodology used in this paper. Section 4 presents the exploratory 
study we conducted on the Mobile Personal Emergency Systems (MPERS). Section 5 presents literature works most related to the focus of our work. In Section 6]we conclude our work and present future directions we envision.

\section{Background}

In this section we present a brief background needed for the understanding of our approach: Contextual Goal Models and Personas.

\subsection{Contextual Goal Models}

Contextual Goal Model (CGM), proposed in [1], extends classic Goal Models by the explicit presentation of the relationship between a goals and their achievement strategies and quality of those strategies on one hand, and the dynamic nature of the system surrounding, i.e. its context, on the other. Context is defined as a partial state of the world in which the system operates and is relevant to its goals [1]. It is a reification of that system surrounding in terms of concrete conditions [11]. Context and its different status operate as an adaptation driver when deciding the goals to activate and the alternatives to adopt and reach the activated goals. It also plays role in deciding the quality of those alternatives, i.e. their contributions to soft-goals. A context may be a patient's health status, a person's relationship status, a specific season of the year, etc.

The CGM presented in Figure 1 depicts the goals to be achieved by a Mobile Personal Emergency Response System (MPERS) which is meant to respond to emergencies in an assisted living environment. The root goal is respond to emergency", which is performed by the actor Mobile Personal Emergency Response. The root goal is divided into four sub-goals: emergency is detected", [p] is notified about emergency", central receives [p] info" and medical care reaches $[\mathrm{p}]$ ([p] stands for patient). Such goals are then further decomposed, within the boundary of an actor, to finally reach executable tasks or delegations to other actors. A task is a process performed by the actor and a delegation is the act of passing a goal on to another actor that can perform it.

\subsection{Persona Characterization}

Persona is a fictional character, an archetype of a group of people of the real world [5]16]. It is a design technique widely used on product development. A persona is defined primarily by its objectives, determined in a process of successive refinements during the initial investigation of the domain of an activity. The Persona structure is derived through a research process, which aims to collect information from various users of the system and, from this, create representative profiles for a group of users. Our persona, Mary Collins in Figure 2, contains the characteristics: age, profession, attributes and goals, but that amount of attributes is not fixed, it depends on how detailed and fine-grained a persona description needs to be which is also dependent on the use and other design artefacts personas are intended to complement. 


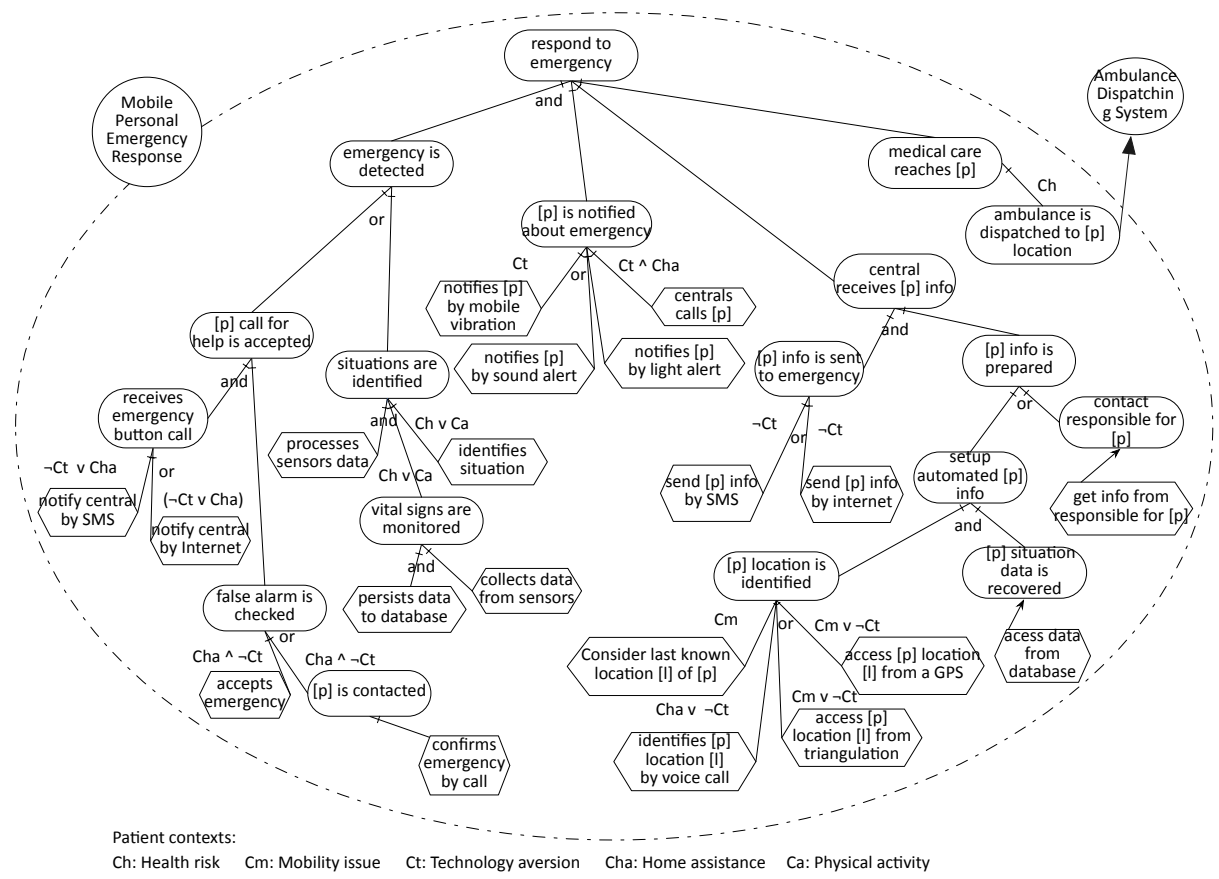

Fig. 1: A CGM for responding to emergencies in an assisted living environment, adapted from [15].

\section{Persona-based Modelling for GORE}

In this section, we present our conceptual model and the methodology of using personas in conjunction with CGM to add human-centred contextual facets to the requirements model.

\subsection{Persona for Contextual Goal Models}

In this section we propose an extension to the CGM integrating it with personas. CGMs take into consideration contexts as conditions on i) the options embedded in the system to be developed, i.e. functional requirements modelled as goals and their strategies, and ii) their quality modelled, i.e. non-functional requirements modelled as soft-goals. Attributes of the users that will actually interact with the system can be seen also as contextual conditions. By considering them at the goal level, usually used at the early stages of development, we add a human perspective early on in the development process. Our extension to the model is highlighted on Figure 3. By including personas to our modeling, we can cater for the actual potential user groups of the software, allowing better specification of the user needs in the software, making the generic goals of the actors more assertive and more specific. In addition, the variability space presented through the space of personas representing users diversity allows identification of the 


\section{Mary Collins (Persona 1)}

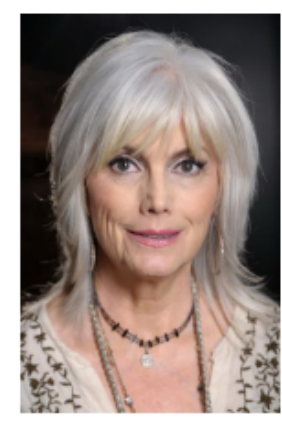

Age: 70 years

Profession: Retired

\section{Attributes}

- Live alone in a small house;

- Does not have a houserkeeper, only diarist every 15 days;

- Does other household chores;

- Fell at home once, but did not fracture any bone;

- Has osteoporosis type 2 at an early stage;

- Has diabetes, high blood pressure and heart problems;

- She is diurnal but wakes up twice at night to go to the bathroom;

- Has 2 childrens who lives in their homes;

- Don't have Wi-Fi at home.

\section{Goals}

- To avoid frustating experiences with technologies;

- To not to worry with her children;

- To feel safe by not falling down at home;

- To have quality of life.

Fig. 2: The characterisation of persona Mary Collins.

impacts to the model to be used on properties like fitness to capabilities and usability. This means that the objectives of the persona can be checked against the objectives and options defined for the product to be developed, in this case the goal model of the system. Therefore, it is possible not only to define the system's functionalities but also to prioritize them from the point of view of the user, who is the one who interacts with the system. Our conceptual model depicted in Figure 3 extends original definitions of the CGM, as our context definition is based on the persona's goals and attributes in its entirety. Major concepts of our model are: Goals, Persona, Context, Attributes, World Predicates, Facts ( $a$ world predicate $F$ is a fact for an actor $A$ iff $F$ can be verified by A. [1]). The CGM contains a set of Contexts linked to its variation points. A Context can aggregate a set of World Predicates in a form of a logical formula and will be validated through a specific Persona. A Persona aggregates a set of Goals and Attributes. Attributes are associated with a set of contextual facts that are aggregated into the World Predicates which constitute the atomic propositions of a certain context.

\subsection{Methodology}

Our methodology comprises the following major steps: structuring personas attributes as contextual facts, characterizing personas contexts, mapping personas contexts into the CGM and checking the achievability of the CGM goals. We should note that our approach brings benefits to the process of goal-oriented requirements modelling inde- 


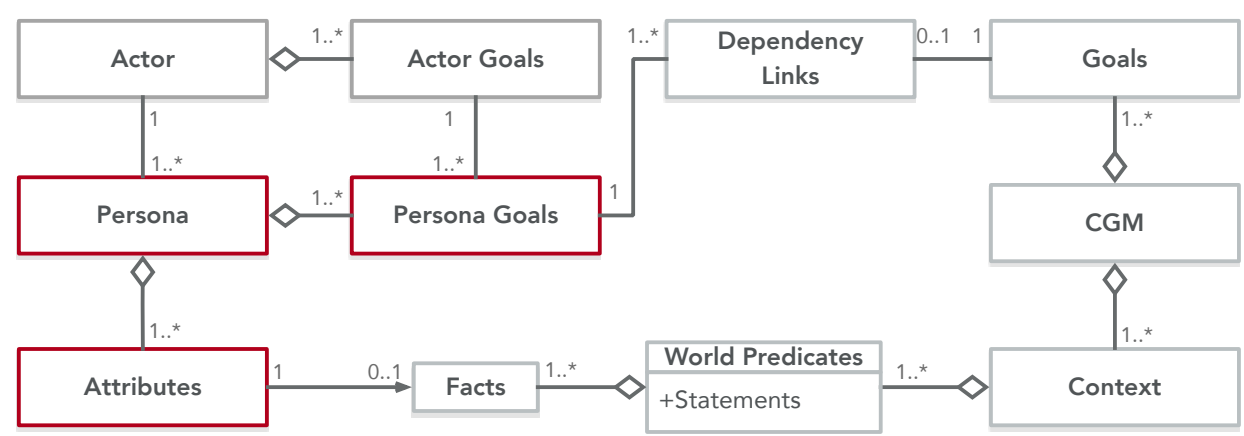

Fig. 3: Conceptual model of our CGM-based persona modelling approach.

pendently from the technology used, i.e. KAOS, $i^{*}$, Tropos. We explain such steps in the following sections.

Structuring Persona Attributes as Contextual Facts The creation of personas relies on information gathered in the early requirements phase. Thus, the determination of the number, attributes, goals and types of personas depends on the potential stakeholders and the elicited requirements and purpose statement of the system. Prior to exploring how personas should be systematically analysed following our methodology, we first define how the informational content of a persona should be first mapped as contextual facts that can be integrated to the CGM [1]. In our work, we argue that persona attributes should be modelled as contextual conditions instead of functional requirements directly mapped as part of those sets of services a system should fulfill, in accordance with the argument in [17]. As such, we model each persona attribute as a contextual fact: $a$ world predicate for an actor $A$ if the fact can be verified by $A$ [1]. We should note that the world predicate is a formula of logic predicates that specifies a context. In our work, the actor is archetyped as personas.

We formalize the description of the persona attributes into contextual facts as follows:

1. $i$ is the id of the persona in the population of interest.

2. $A_{i} \in\left\{A_{1}, A_{2}, \ldots, A_{n}\right\}$, where $\mathrm{A}$ is a set of attributes as nominal categorical variables of $i$.

3. Each attribute $A_{i}$ may have a corresponding contextual fact $F_{j}$, where $i \leq j$.

4. $i=\bigcup_{n=1}^{j} F_{n}$, the persona $i$ is characterized as the union of $F_{j}$ contextual facts.

Following the work of Chapman et al. [4], the persona attributes can be sorted as a key-value list, where each key is a nominal categorical variable instantiated by an attribute of the persona. For example, from the excerpt of the persona Mary Collins, we can have a list of attributes: $A_{1}$ ("Mary Collins") \& $B_{1}$ ("high probability of fall incidents") \& $C_{1}$ ("unfamiliar with technology") \& etc, , where 1 is the id that uniquely identifies the persona Mary Collins. Attributes $A_{1}, B_{1}$ and $C_{1}$ are categorical variables 
that could be accordingly mapped to contextual fact such as name, healthProblem and techAversion, respectively.

In the formalization of personas attributes above, note that $F_{j}$ is an index function that characterizes the facts under study pertaining all personas. For a particular category variable, the purpose is to have the more variation as possible to significantly represent the target system. For example, in order to comprehensively represent a group of patients to be modelled for the MPERS, various types of illness and health risk may be represented in different persona representations.

Characterizing Persona Contexts Once the persona's contextual facts are properly characterized, we then proceed to characterize all the contexts which will be further instantiated in the CGM to analyse if the system goals will be affected by the persona's characteristics as contexts of operation. To characterize the context that will be triggered by the persona we take in consideration the semantic information of the contextual facts. For example, in our persona Mary Collins the attribute $B_{1}$ : "high probability of fall incidents" mapped into contextual fact healthProblem can take part of the context healthRisk. Other facts could compose such context if, for example, another persona is characterized with another healthProblem fact that could fit into healthRisk context.

Following the work in [1], we specify context as a predicate formula of and/or combinations of statements and facts. Note that the contextual facts in our work map only those relevant persona information that can be directly verified through data gathering, for example. In Figure 4 we model context $C h$ : healthRisk. Such context is defined by the following predicate formula: $f 1 \vee f 2 \vee f 3 \vee f 4 \vee f 5 \vee f 6 \& w p 1$. Therefore, context $C h$ applies if at least one of such contextual facts is true (present) in the modelled personas and the health status of the patient is characterised either as low $(f 7)$, medium $(f 8)$ or critical $(f 9)$ status. In particular, for other fuzzy typed values, an analogous modelling can be carried out as characterised by $w p 1$, which actual range values should be defined by the domain expert. We should note that it is out of scope of our current work to define an approach to elicit contexts. We assume that the contexts characterization into facts should rely on domain expert validation. However, one could also use other sources of information to extract relevant contexts from available information, if present, or conduct a data gathering of personas' relevant information via survey study following the work of Chapman et al. [4].

In Figure 1 we illustrate five different patient contexts in the MPERS case study, where each context variant characterizes their own context formula. Namely, such contexts are the following ones: $\mathrm{Ch}$ :health risk, $\mathrm{Cm}$ : mobility issue, $\mathrm{Ct}$ : technology aversion, Cha: home assistance and Ca: physical activity. Once the contexts related to the persona are identified, the next step consists of the mapping of such contexts into the CGM. The contexts identified for the patients of the MPERS case study are presented in Figure 1 Once the contexts (formula) of the CGM have been defined, it is then possible to define all the contexts that are triggered by a given persona. Given all those contextual facts of a persona, if a context formula evaluates to true for the facts of a persona, it means such context is part of the set of contexts triggered by a persona. We formalize such set definition as follows: 


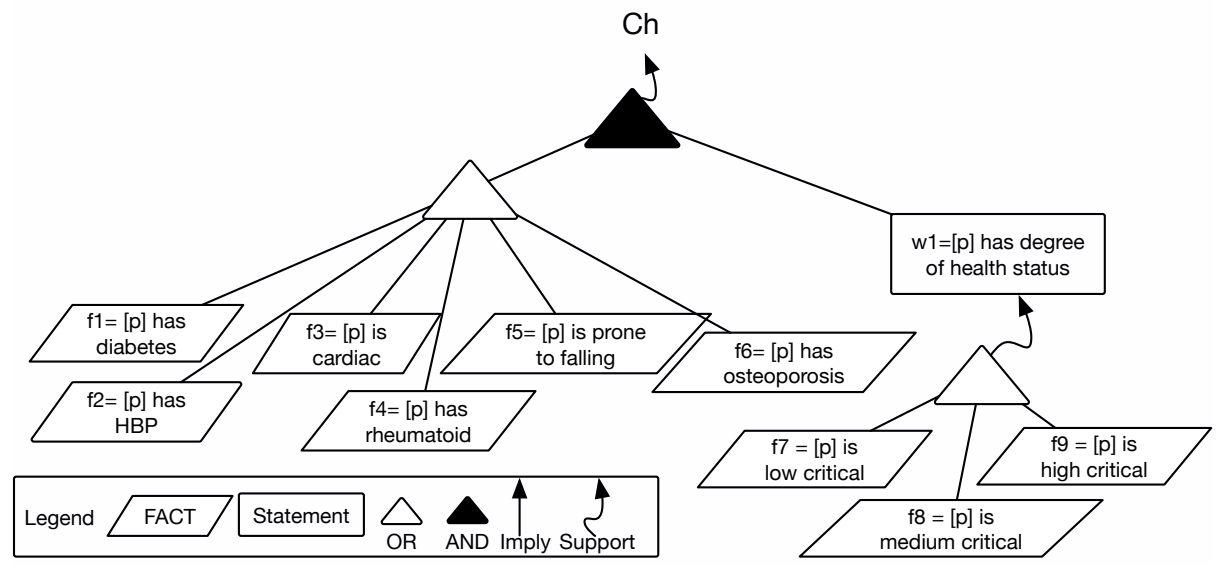

Fig. 4: Context modelling excerpt.

Definition 1 (Persona Context Set). Let the mapping function $C: i \stackrel{C j}{\longrightarrow}\{T, F\}$ which returns true or false for the facts of persona $i$ applied to context $C_{j}$. If $C_{j}(i)=T$, it means that $C_{j} \in \Omega$, where $\Omega$ is the set of contexts triggered by persona $i$.

In case of our persona Mary Collins, she triggers the context set $C h, C h a$ and $C t$. The trigger of Mary's $C h$ is due to the fact that at least one of the facts from $f 1$ to $f 6$ are part of Mary's context facts list. Actually, not only $f 1$ but also $f 2, f 3$ and $f 6$ are facts of the persona Mary Collins. While $C t$ is triggered by the fact she is not technology friendly ( $f 19)$ and Cha is triggered by the fact she has an assisted living device ( $f 14)$.

Structuring Persona Goals Due to the fact that a persona represents user groups by using a rich and highly memorable description, they are easy to understand during the whole development process. In our proposal, a persona has several goals to achieve by using the system. The goals of a persona can be described in a holistic manner using the persona's psychological characteristics, attitudes, motivations, and preferences. Therefore, persona goals encapsulate a comprehensive description of representative users' needs and expectations regarding the system under development. It also clarifies decision rationales to prioritize a particular goal. Initially, during the definition of a persona as described in Figure 2, the requirements team should textually describe the persona attributes and goals. Following the proposal by [9], the persona attributes and goals previously defined in a textual format can be represented by means of goals, softgoals, or tasks. As such, an actor can be associated by different personas, in which each persona has his/her specific goals and contexts, as illustrated in Figure 5. For instance, in our case study, actors can be patients and doctors. The group of patients as users is very heterogeneous and can be classified in specific personas such as: Mary who is averse to technology, Dorothy who has difficulty in walking, and Jennifer who does physical activity, has facility with technology but takes controlled medication. In our study, the 
actor doctor is represented by the persona Pau 4 Then, we identify the extent to which

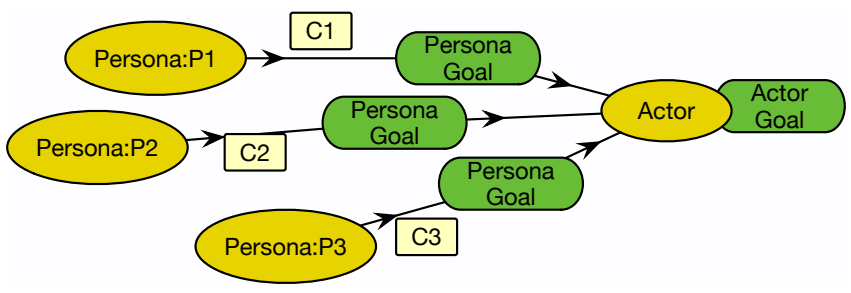

Fig. 5: The Relationship Between Actors and Persona Goals

each element contributes to the persona goal satisfaction. The persona goals have relationships with the CGM via dependency links. Therefore the satisfiability of the persona goals can be defined as follows:

Definition 2 (Persona Goal Satisfaction). Let the context set $\Omega$ triggered by persona $i$, the actor goal $\Gamma$, which the persona goal is link dependent, and the target system $C G M$. The persona goal satisfaction property $\Phi_{i}$ is achieved when $(\Omega, \Gamma, C G M) \vDash \Phi_{i}$

For example, in the MPERS scenario, the patient actor they want to be assisted. While the actor representing the medical doctor they want to assist the patient. However, it not only suffices to know if their goal to either be assisted or to assist via the MPERS will be achieved. The context of the patient and the doctor also needs to be taken into consideration to make sure the persona instantiating the corresponding actors will have their goals satisfied. Therefore, the need to know the persona context is paramount to learn if the CGM goal will be reached. So the satisfaction of the persona goal in the MPERS means that the root goal of the CGM was satisfied under the persona context set (e.g. Mary's context set) and its actor goal (e.g. be assisted via MPERS).

Goal Achievement Check In our work, the persona goal satisfaction is carried out through the goal achievement check algorithm, where we leverage the achievability of goals in a CGM by adding the human perspective through the person context. Such expressiveness will enable richer adaptation decisions that not only consider the static achievability but also the achievability where the user context can be explicitly modelled and its effect on the fulfillment criteria of a goal. The achievability of a goal and the space of adoptable alternatives to achieve it are essential information to plan adaptation, seen as a selection and enactment of a suitable alternative to reach a goal under a certain persona context criteria.

In Algorithm 1 we evaluate the achievability of the system goals under the contexts triggered by the persona. The algorithm has as input information the Contextual Goal Model and the persona context facts. The algorithm is recursive, building on the fact that the CGM is a tree-structured model and that each refinement may be seen as a tree

\footnotetext{
${ }^{4}$ Such personas are available in the GitHub link for this paper: https://github.com/
} CJTS/REFSQ_2018 
node. The Algorithm considers the root node of the CGM (line 1). Given the context facts that characterise the persona and a set of logical relations between the context variables, in line 2, the call to getContextSet method returns the context sets triggered by the persona to check if there exists a truth assignment for all variables that makes the conjunction of the persona context formula satisfiable. If such assignment exists, then the formula is satisfiable, otherwise it is unsatisfiable under the assumed logical relations.

After that, the contextual goal model is traversed considering the mapped persona context set and the tree structure of the CGM. The algorithm checks whether the goal node is itself applicable under the current persona context (line 3), returning NULL if it is not (line 4). In the particular case when the node type is a task (6) it can decide on the goal achievability and returns a plan consisting only of such task (line 9). If the node is not a task, the Algorithm starts defining an execution plan that fulfills the persona context set (line 11). For each of the applicable refinements (line 13), it will evaluate if it is achievable (line 14p. If the refinement is achievable then, for OR-decompositions, the algorithm returns this plan immediately (line 17) and for AND-decompositions it is added to the complete plan (line 20). If the CGM node is not achieved the algorithm returns the root node of the failed subtree (lines 22- 25). As such it means that in the fulfilment of that particular failing CGM node, better alternative strategies need to be addressed so that frustrations with the actual user do not happen under real life conditions. Finally, for AND-decompositions, should all refinements render achievable it will return the complete execution plan for the persona contextual information (line 28).

\section{Feasibility Study}

To evaluate our method, which mainly formalizes the information of personas and map them as contextual requirements, we implemented the Algorithm proposed in Section 3.2 and applied it in the MPERS CGM. The algorithm analyses the achievability of goals in a CGM when considering a certain persona and their attributes as contexts. The evaluation was designed according to the Goal-Question-Metric (GQM) framework [19] presented in (Table 11). For this purpose, we evaluated the efficiency of the algorithm, run it to test the achievability of goals and the planning it yields for each modelled personas with the varying attributes and goals. The purpose was to provide a proof of concept and evidence for feasibility.

\subsection{Experiment setup}

The study consisted in evaluating the methodology in the MPERS case study. We used the goal model provided in Figure 1 We modelled four personas where three of them are patients (Mary, Jennifer and Dorothy) and one of them is a medical doctor (Paul). For the patients, we applied the five contexts modelled in Figure 1 . As for the medical doctor we applied three new contexts: Cc: means of communication, Ci: means of information sharing, Che: means of assisting. The evaluation was based on a prototype implemented in C\#. The experiments were executed on an Intel(R) Core i5, $1.6 \mathrm{GHz}$ and $4 \mathrm{~GB}$ of RAM. For the sake of space, we do not report all the detailed information 


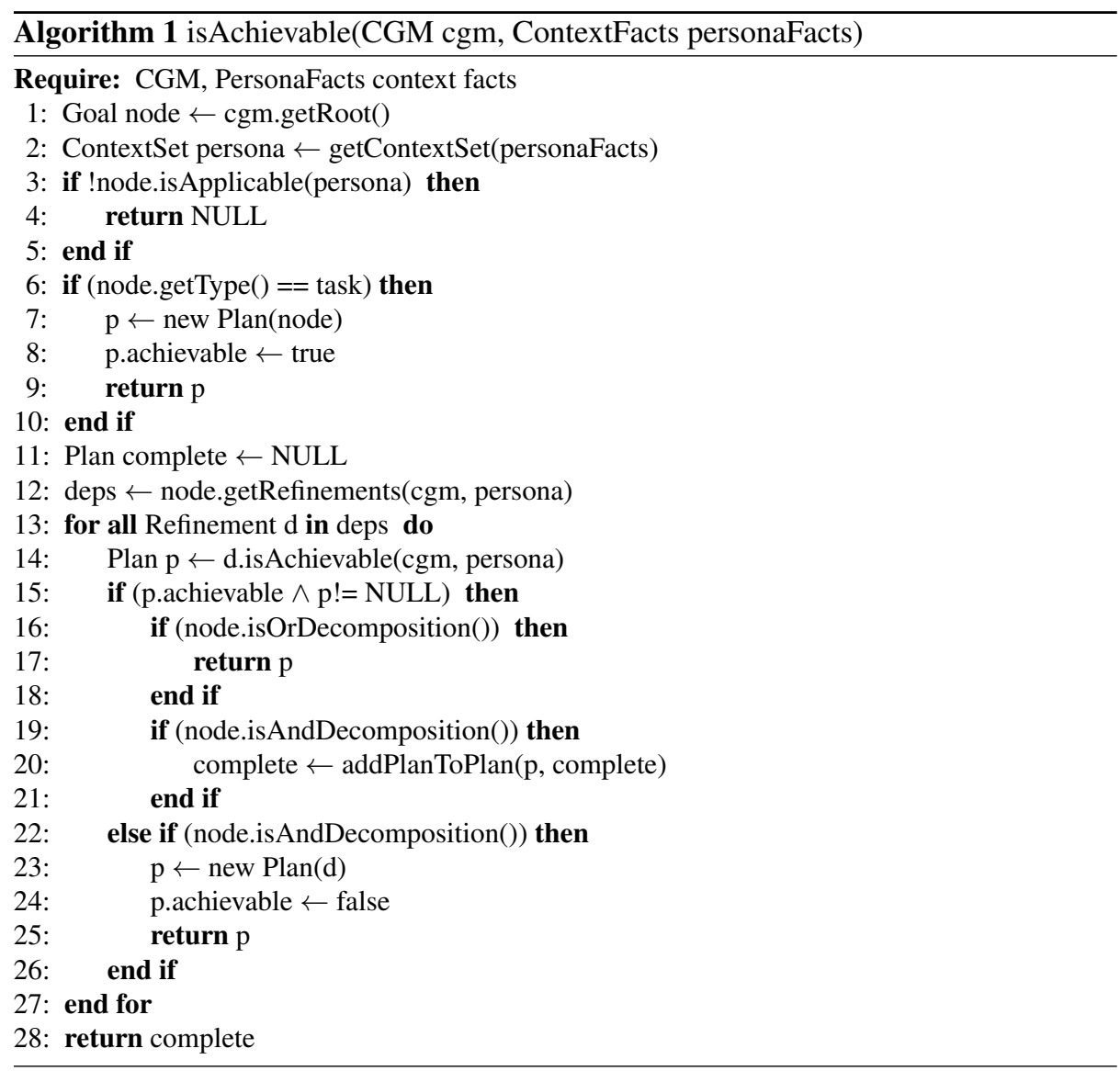

of our feasibility study and the implementation details, but they can be accessed via our provided Github repository link. ${ }^{5}$

\subsection{Results}

Question 1: Is the algorithm efficient to come up with an execution plan? - We evaluated the time for the algorithm to come up with an execution plan considering the MPERS CGM in Figure 1 for the four modelled personas. The results showed that the algorithm took at most $40.10^{-3} \mathrm{~ms}$ (40 microseconds) to come up with an answer for each persona context. Therefore, the algorithm can be considered quite efficient for setup analysed.

Formally speaking, the algorithm can be proven as linear time complexity $O(n)$, where $n$ is the number of nodes in the CGM. The major complexity of the algorithm is in the execution of each Refinement $d$ in deps (lines 13 27). The algorithm recursively invokes itself for the CGM of the Refinement (sub goal, task or delegation). Since this

${ }^{5}$ Source code, goal model and evaluation of our approach are available at: https:// github.com/CJTS/REFSQ_2018 
Table 1: GQM devised plan

\begin{tabular}{|l|l|}
\hline \multicolumn{2}{|c|}{ Goal: Analysis of the achievability of the goals } \\
\hline \multicolumn{1}{|c|}{ Question } & \multicolumn{1}{|c|}{ Metric } \\
\hline $\begin{array}{l}\text { Q1. Is the algorithm efficient to come up } \\
\text { with an execution plan }\end{array}$ & Execution time \\
\hline $\begin{array}{l}\text { Q2. Does the algorithm allow testing } \\
\text { and explaining persona-based goal } \\
\text { achievability? }\end{array}$ & Yes/No \\
\hline $\begin{array}{l}\text { Q3. Are the plans provided by the } \\
\text { algorithm correct? }\end{array}$ & $\begin{array}{l}\text { O of correct } \\
\text { plans }\end{array}$ \\
\hline
\end{tabular}

invocation is performed on trees of height lower or equal to the CGM tree we can consider that the root node has $n$ refinements and this is performed in $O\left(n_{k}\right)$ time, where $n_{k}$ is the amount of nodes in the $k$ sub tree of node $n$. Then, each node in the sub tree is visited exactly once. Therefore, the time to visit all $n$ nodes is: $O(n)+O\left(n_{k}\right)$. That amounts to $O(n)$, since summing across the number of children of each tree node is equivalent to summing over all nodes. We should note that, in the particular case that the root goal is not applicable the algorithm simply returns NULL in constant time, thus $O(1)$. Likewise, the call to the getType method returns in constant time (lines 39 .

Question 2: Does the algorithm allow testing and explaining persona-based goal achievability? - We considered 19 distinctive facts for the considered personas. Such facts can be distributed in eight distinctive contexts: five for the patients and three for the medical doctor, as previously mentioned in the Study Setup and further explained in the Github link of this work. Having the root achieved for each persona means that the root goal of the MPERS' CGM is achieved and, therefore, considering the persona context, the system is able to meet its goals. Out of the four modelled personas, only Mary did not have the MPERS goals achieved. It happened due to the fact that Mary has technology aversion to some degree since she fears having frustrating experiences with technology (context fact $f 19$ ). Such fact triggers context $C t$ and hinders the goal fulfilment that the central receives $[p]$ info since its left subtree will be fulfilled if the patient does not have technology aversion. As a result, alternative solutions need to be devised (and included in the MPERS CGM) particularly related to the $[\mathrm{p}]$ info is sent to emergency goal, where people with technology aversion, like Mary Collins, may be able to guarantee their information is sent under an emergency situation without requiring them to be technology friendly.

Question 3: Are the plans provided by the algorithm correct? - Out of the four modelled personas, the algorithm provided two execution plans as presented in Figure $6 \mathrm{We}$ represent the two plans as one, where they differ on the highlights in red boxes. The activity in the red box labelled Planning 1 corresponds to the available execution plan that is achieved in the context of Dorothy (patient) and Paul (medical doctor). While the activity in the red box labeled Planning 2 corresponds to the available execution plan that is achieved in the context of Jennifer (patient). Note that the fact that Dorothy and Paul share the same planning does not mean they trigger the same contexts since their context sets are disjunctive. On the other hand, considering that Jennifer and Dorothy are both patients, they trigger different execution plans. While Dorothy has some difficulty 
in walking and therefore considered as having some problem on her ability to move $\mathrm{Cm}$. In case she falls and her location identification is needed, the system will Consider last known location of Dorothy. The variability that the planning captured is exactly what has been conceived for those patients with mobility issue in the CGM devise. Therefore, the plans provided by our algorithm are correct.

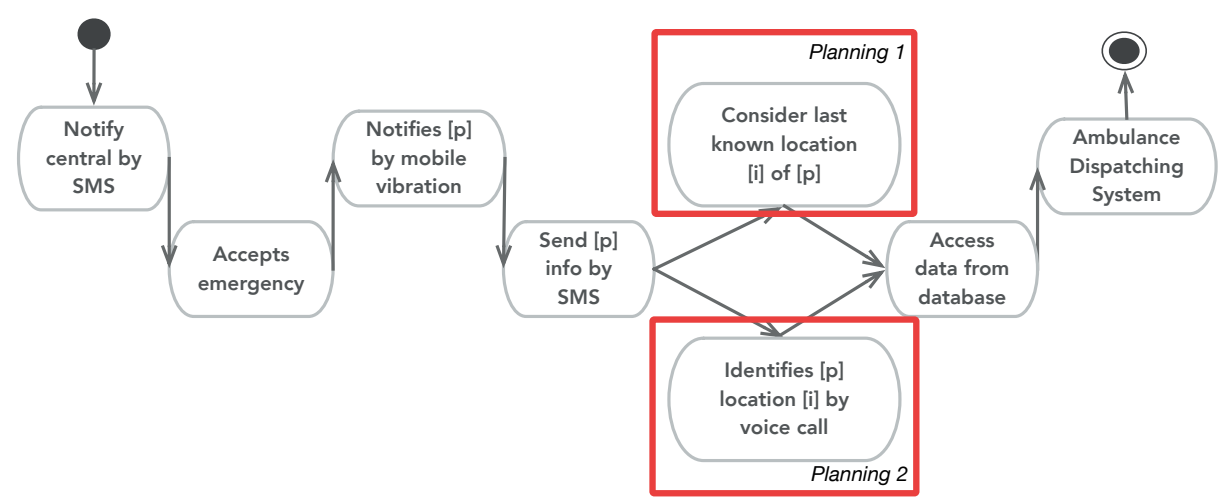

Fig. 6: Achievable Plans for the provided personas contexts.

\section{Related Work}

Several works studied Persona in Software Engineering context [2]10|12]3]. Haikara [12] tackles the subject in agile software development where the argument is that agile software development methods do not seem to address usability and interaction design issues enough and the author proposes an extension on the interaction design process by using personas. Castro et al. [3] use the personas technique and integrate it into the requirement analysis activity. They advocate the necessity to understand users who interact with the system. The work of Faily and Lyle [10] illustrates how personas can be integrated into software tools to support usability and software engineering. That works presents guidelines that software engineering tools should incorporate to support the design and evolution of personas. By using personas in the requirement analysis phase they can gain a better understanding of the user and can improve the usability of the system. Chapman et al. [4] propose a formal model to understand persona information in terms of factual attributes. They use such information to guide the extraction of a comprehensive variety of personas. In our work, we take personas a step further and embed them in the system requirements model so that we allow a more formal and automated support to the alignment between users diversity and the system at the early stage of goals and intentionality.

The works of [7]8] amongst the first to address personas in the context of a goal modelling process. They use the goals model to visualise and help validate personas. While in [7] the author is concerned with usability issues, in [8] the authors are concerned with trust issues. In our work, we use personas information as contextual facts 
and the impact personas-derived contexts will have in the goal model the personas might be embedded in. The work of Di Francescomarino et al. [6] tackles personas in the context of goal modelling and uses the User-Centered Design (a series of well-defined methods and techniques that comes from social sciences) towards that integration. Their work aims at defining a modeling framework that integrates the goal-oriented paradigm, process modeling and User-Cantered design techniques and methods to capture the intentional elements of the user (e.g. goals, preferences, assets, etc.).

The work in [1] proposes an explicit notion of context and its relation to requirements and applies that on goal model providing Contextual Goal Models (CGM). There are few works that adopt CGM as modelling baseline such as Guimaraes et al. [15] and Mendonca et al. [14]. The work in[15] uses the concept of Pragmatic Goals to enhance the contexts of CGMs. Pragmatic Goals are the idea that a goal's interpretation varies according to context. While the work in [14] uses the CGM structure to provide quantitative dependability analysis by means of probabilistic model checking. Despite the benefits of their approach, they do not cater primarily for the human perspective which could hinder altogether their analysis processes in that aspect.

\section{Discussion, Conclusion and Future Work}

The use of persona as an enabling technique to personalize and add a human-centric aspect to business requirements was proposed. The premise is that this would make the system analysis closer to its personnel both in relation to their job role description and also unique personalities. While personas are usually used in the literature of $\mathrm{HCI}$ and usability, we argued that they can be equally useful at the early stages of requirements engineering including the intentionality and strategic interest modelling done via languages like goal modelling. Personas in this case are not only about how the users use the system typically but also about the alignment between their intentions and capabilities on one hand and the socio-technical solutions on the other.

Concerning the efficiency of our approach, we have shown the algorithm works in $\mathrm{O}(\mathrm{n})$ complexity. Therefore, the number of personas to be analysed would not be a computational issue. As for the optimal number of personas one should take into account, Chapman et al. [4] have already proposed an ideal number of personas previously. Nevertheless, the higher the number of the personas, the more complex to map their information into the goal model. On the hand, such complextity stems from the mapping of contexts into goals following the CGM background approach. As for the application domain benefit, all those that benefit from a goal-oriented modelling approach could benefit from our work, if the human perspective should be taken into account as any socio-technical system would require.

We recognize that a better management of our approach requires a more elaborated conceptualization of personas. This includes the distinction between different families of personas characterizers and relations amongst them and goals. Remarkable classes of these attributes include capabilities, personality traits, collaborative and competitive nature, intrinsic and extrinsic motivation and learning styles. This should also be mapped to goals for fitness and alignment. For example, a business goal which requires heavy dependency and interaction with other actors to achieve would not fit a persona who is introvert in personality and prefers well-defined tasks and less tolerant to deviation 
from the norm. In addition, personas goals could be of various families as well including social recognition, values, promotion goals, etc. which are aimed to be achieved in tandem with the business goals but not part of the contractual settings.

For future work, as a process of specification we may need to think of two parallel processes to allow a separation of concerns. The first strand would concern the business requirements modelling while the other looks at the personnel and their expectations and requirements in the first instance. This would enable independence before the triangulation stage would start. Such a mixed-method could be applied in an iterative and evolutionary style where argumentation and negotiation can be enabled. Participatory design principles and processes would be a potential fit. In summary, a human-centred approach to requirements modelling would also benefit from similar approaches adopted at the design stage but adapted to the level of abstraction and emphasis of requirements modelling. Additionally, a real-life case study will be addressed.

\section{Acknowledgment}

The authors would like to express their gratitude for the fruitful discussions with Shamal Faily, Felipe Pontes, Célia Ralha and Renato Pina during the development of this work. Genaína would like to thank CAPES-PROCAD for the partial financial support of this work.

\section{References}

1. R. Ali, F. Dalpiaz, and P. Giorgini. A goal-based framework for contextual requirements modeling and analysis. Requir. Eng., 15(4):439-458, Nov. 2010.

2. M. Aoyama. Persona-and-scenario based requirements engineering for software embedded in digital consumer products. In Requirements Engineering, 2005. Proceedings. 13th IEEE International Conference on, pages 85-94. IEEE, 2005.

3. J. W. Castro, S. T. Acuña, and N. Juristo. Integrating the personas technique into the requirements analysis activity. In Computer Science, 2008. ENC'08. Mexican International Conference on, pages 104-112. IEEE, 2008.

4. C. N. Chapman, E. Love, R. P. Milham, P. ElRif, and J. L. Alford. Quantitative evaluation of personas as information. In Proc. of the Human Factors and Ergonomics Society Annual Meeting, volume 52, pages 1107-1111. SAGE Publications, CA, 2008.

5. A. Cooper et al. The inmates are running the asylum:[Why high-tech products drive us crazy and how to restore the sanity].

6. C. Di Francescomarino, C. Leonardi, A. Marchetto, C. D. Nguyen, N. A. Qureshi, L. Sabatucci, A. Perini, A. Susi, P. Tonella, and M. Zancanaro. A bit of persona, a bit of goal, a bit of process... a recipe for analyzing user intensive software systems. In $i$ Star 2010 Proceedings of the 4 th International $i *$ Workshop, page 36. Citeseer, 2010.

7. S. Faily. Bridging User-Centered Design and Requirements Engineering with GRL and Persona Cases. In Proc. of the $5^{\text {th }}$ International $i^{*}$ Workshop, pages 114-119. CEUR Workshop Proceedings, 2011.

8. S. Faily and I. Fléchais. Eliciting and Visualising Trust Expectations using Persona Trust Characteristics and Goal Models. In Proc of the $6^{\text {th }}$ International Workshop on Social Software Engineering, pages 17-24. ACM, 2014. 
9. S. Faily and I. Flchais. Eliciting and visualising trust expectations using persona trust characteristics and goal models. Proc. of the 6th International Workshop on Social Software Engineering, 2014.

10. S. Faily and J. Lyle. Guidelines for integrating personas into software engineering tools. In Proceedings of the 5th ACM SIGCHI symposium on Engineering interactive computing systems, pages 69-74. ACM, 2013.

11. A. Finkelstein and A. Savigni. A framework for requirements engineering for context-aware services. IEEE Computer Society Press, 2001.

12. J. Haikara. Usability in agile software development: extending the interaction design process with personas approach. In International Conference on Extreme Programming and Agile Processes in Software Engineering, pages 153-156. Springer, 2007.

13. F. Long. Real or imaginary: The effectiveness of using personas in product design. In Proc. of the Irish Ergonomics Society Conference, pages 1-10. Irish Ergonomics Society, 2009.

14. D. F. Mendonça, G. N. Rodrigues, R. Ali, V. Alves, and L. Baresi. GODA: A goal-oriented requirements engineering framework for runtime dependability analysis. Information \& Software Technology, 80:245-264, 2016.

15. F. Pontes Guimaraes, G. Nunes Rodrigues, D. Macedo Batista, and R. Ali. Pragmatic Requirements for Adaptive Systems: A Goal-Driven Modeling and Analysis Approach, pages 50-64. Springer International Publishing, Cham, 2015.

16. J. Pruitt and J. Grudin. Personas: Practice and theory. In Proceedings of the 2003 Conference on Designing for User Experiences, DUX '03, pages 1-15, New York, NY, USA, 2003. ACM.

17. A. Sutcliffe, S. Fickas, and M. M. Sohlberg. Personal and contextual requirements engineering. 13th IEEE International Conference on Requirements Engineering (RE'05), pginas: 19-28, 2005.

18. A. Van Lamsweerde. Goal-oriented requirements engineering: A guided tour. In Requirements Engineering, 2001. Proceedings. Fifth IEEE International Symposium on, pages 249262. IEEE, 2001.

19. R. Van Solingen, V. Basili, G. Caldiera, and H. D. Rombach. Goal question metric (gqm) approach. Encyclopedia of software engineering, 2002.

20. E. Yu and J. Mylopoulos. Why goal-oriented requirements engineering. In Proceedings of the 4th International Workshop on Requirements Engineering: Foundations of Software Quality, volume 15, pages 15-22, 1998. 